Published in final edited form as:

Biochemistry. 2020 February 04; 59(4): 627-634. doi:10.1021/acs.biochem.9b00971.

\title{
X-ray crystal structures of the influenza M2 proton channel drug- resistant V27A mutant bound to a spiro-adamantyl amine inhibitor reveal the mechanism of adamantane resistance
}

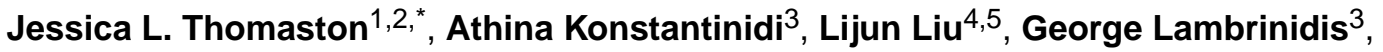

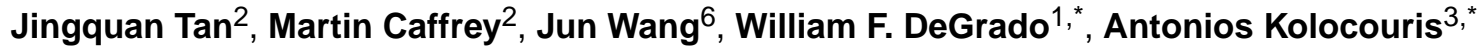

${ }^{1}$ Department of Pharmaceutical Chemistry, University of California, San Francisco, CA 94158, USA. ${ }^{2}$ Membrane Structural and Functional Biology (MS\&FB) Group, School of Medicine and School of Biochemistry and Immunology, Trinity College Dublin, Dublin D02 R590, Ireland. ${ }^{3}$ Department of Pharmaceutical Chemistry, National and Kapodistrian University of Athens, 15771 Athens, Greece. ${ }^{4}$ State Key Laboratory of Chemical Oncogenomics, School of Chemical Biology and Biotechnology, Peking University Shenzhen Graduate School, Shenzhen 518055, China. ${ }^{5}$ DLX Scientific, Lawrence, KS 66049, USA. ${ }^{6}$ Department of Pharmacology and Toxicology, College of Pharmacy, University of Arizona, Tucson, Arizona 85721, USA.

\section{Abstract}

The V27A mutation confers adamantane resistance to the influenza A matrix 2 (M2) proton channel and is becoming more prevalent in circulating populations of influenza A virus. We have used X-ray crystallography to solve structures of a spiro-adamantyl amine inhibitor bound to M2(22-46) V27A and also to M2(21-61) V27A in the Inward closed $_{\text {conformation. The spiro- }}$ adamantyl amine binding site is nearly identical for the two crystal structures. Compared to the M2 "wild type" (WT) with valine at position 27, we observe that the channel pore is wider at its $\mathrm{N}$-terminus as a result of the V27A mutation and that this removes V27 side chain hydrophobic interactions that are important for binding of amantadine and rimantadine. The spiro-adamantyl amine inhibitor blocks proton conductance in both the WT and V27A mutant channels by shifting its binding site in the pore depending on which residue is present at position 27. Additionally, in the structure of the M2(21-61) V27A construct, the C-terminus of the channel is tightly packed relative to the M2(22-46) construct. We observe that residues Asp44, Arg45, and Phe48 face the center of the channel pore and would be well-positioned to interact with protons exiting the M2 channel after passing through the His37 gate. A 300 ns molecular dynamics (MD) simulation of

\footnotetext{
*Corresponding Authors: william.degrado@ucsf.edu, jessica.thomaston@gmail.com, ankol@pharm.uoa.gr. Author Contributions

All authors have given approval to the final version of the manuscript.

ASSOCIATED CONTENT

The Supporting Information is available free of charge on the ACS Publications website.

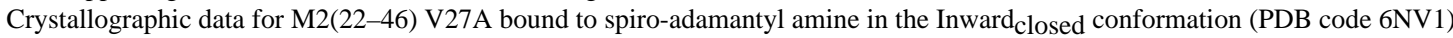
(CIF)

Crystallographic data for M2(21-61) V27A bound to spiro-adamantyl amine in the Inward closed conformation (PDB code 6OUG) (CIF)

Supplemental figures and tables, detailed experimental methods, detailed molecular dynamics methods (PDF)
} 
the M2(22-46) V27A - spiro-adamantyl amine complex predicts with accuracy the position of the ligands and waters inside the pore in the X-ray crystal structure of the M2 V27A complex.

\section{Graphical Abstract}

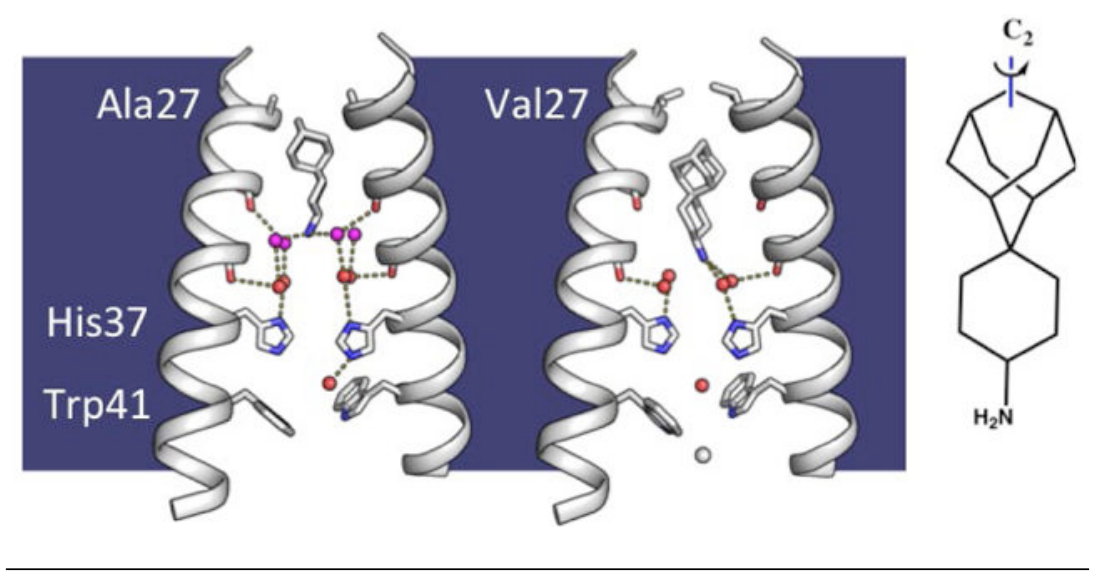

\section{INTRODUCTION}

Influenza A M2 is a multifunctional viral protein whose domains play several roles during the viral lifecycle. It is a homotetramer that contains monomers made up of 97 amino acids. The N-terminal domain (residues 1-22) is well-conserved and plays a role in incorporation of M2 into the virion. ${ }^{1}$ The transmembrane (TM) domain (22-46) forms a unidirectional, proton-selective channel ${ }^{2,3}$ that acidifies the viral interior after the virus is endocytosed into the host cell. This allows viral ribonucleoproteins (vRNPs) to dissociate from the matrix 1 (M1) protein; when proton conductance through M2 is blocked by the adamantane drugs (Fig. S1), this dissociation is prevented and the virus is no longer able to replicate. ${ }^{4}$ The proton channel function of M2 also serves a secondary role in the de-acidification of the Golgi apparatus so that acid-sensitive hemagglutinin is not prematurely activated before the virus assembles and buds. ${ }^{5}$ The minimal construct needed for selective, unidirectional proton transport is the TM domain (residues 22-46). ${ }^{6,7}$ The TM domain is relatively well-conserved compared to the rest of the channel, and only a small number of mutants retain conductance rates similar to the $\mathrm{WT}^{8}{ }^{8}$ The cytosolic helix of $\mathrm{M} 2$ induces membrane curvature and is involved in viral budding and scission, 9,10 and the C-terminal tail interacts with the M1 protein. ${ }^{11}$

In recent years, adamantane drug-resistant mutants have become prevalent in circulating viruses. Some viruses are resistant to the adamantanes and neuraminidase inhibitors, ${ }^{12-14}$ thus highlighting the need to develop new influenza antivirals. The two most prevalent drugresistant mutants are $\mathrm{S} 31 \mathrm{~N}$ and V27A. ${ }^{15}$ While the S31N mutation was present in populations of influenza before the introduction of the adamantane drugs, V27A has become enriched by selection pressure. ${ }^{16}$, Double mutants containing both S31N and V27A mutations have been observed. ${ }^{17,18}$ The percent of influenza viruses containing the V27A mutation varies widely depending on the viral strain and season, with some studies reporting incidence as high as $7-10 \% .19,20$ Overall, the V27A mutation is becoming more prevalent in 
circulating populations of influenza, ${ }^{18}$ so there has been interest in designing drugs to target it. $^{21-26}$

Here we present X-ray crystal structures of the drug-resistant V27A mutant of M2 bound to a spiro-adamantyl amine inhibitor, using both M2(22-46) and M2(21-61) constructs for crystallization trials. Spiro-adamantyl amine was developed by molecular dynamics simulation-directed design and was able to inhibit the conductance of protons in both the V27A mutant and the WT channel in two electrode voltage clamp (TEVC) assays using Xenopus oocytes, with an $\mathrm{IC}_{50}$ of $0.3 \mu \mathrm{M}$ against the V27A channel and an $\mathrm{IC}_{50}$ of $18.7 \mu \mathrm{M}$ against the WT channel (compare to $\mathrm{IC}_{50}=15.7 \mu \mathrm{M}$ for amantadine against the WT channel). ${ }^{26}$ Spiro-adamantyl amine was further shown to have both in vitro and in vivo antiviral activity against influenza A M2 V27A mutant virus. ${ }^{22}$ Therefore, spiro-adamantyl amine appears to represent a promising antiviral drug candidate that warrants further characterization. In this study, we focus on understanding the mechanism of action of spiroadamantyl amine in inhibiting the M2 V27A mutant by solving high-resolution X-ray crystal structures. It was found that when Val27 is mutated to Ala, the diameter of the channel pore at the drug binding site increases and the hydrophobic interactions that stabilize the binding of adamantanes to the WT channel are removed. This widening of the pore at the channel's $\mathrm{N}$-terminus allows the spiro-adamantyl amine compound to bind with its adamantyl group higher in the channel relative to previous structures of drug-bound M2 WT. The inhibitor binds with its ammonium group pointed down the channel pore, in the direction of the gating His 37 residues. The inhibitor ammonium group interacts indirectly with the gating His37 residues through two intervening water layers, compared to one water layer in the M2 WT complex with the same spiro-adamantyl amine inhibitor. This interrupts hydrogen bonded water networks leading down to the His 37 gate and prevents proton conduction through the channel. In the structure of M2(21-61) V27A bound to spiro-adamantyl amine, we observe tight packing at the channel's C-terminus, particularly at residue Arg45.

\section{MATERIALS AND METHODS}

\section{M2 peptide synthesis and crystallization.}

M2(22-46) V27A and M2(21-61) V27A were synthesized using Fmoc chemistry with an optimized solid phase synthesis protocol and purified using reverse phase HPLC. ${ }^{27,28}$ Purity and identity of the peptides were confirmed using analytical HPLC and MALDI-MS. The peptides were reconstituted into the lipid cubic phase (LCP) with modifications to the protocol described by Caffrey and Cherezov. ${ }^{29}$ The peptides were dissolved in ethanol and added directly to dry monoolein powder along with an ethanol stock of spiro-adamantyl amine, then the excess ethanol was removed using a stream of nitrogen followed by lyophilization overnight. ${ }^{27,30,31}$ The cubic phase was formed by melting the sample at $40{ }^{\circ} \mathrm{C}$ and mixing with an aqueous solution containing MNG-3-C8 detergent, ${ }^{32,33}$ which in previous crystallization trials and solution NMR studies has been shown to stabilize the Inward $_{\text {closed }}$ conformation of the M2 channel. ${ }^{34,35}$ Crystallization conditions were screened in plastic 96-well sandwich trays (Laminex) using a LCP Mosquito crystallization robot (TTP Labtech). See the Supporting Information for detailed experimental methods. 
Crystals were diffracted at the Advanced Light Source (Berkeley, USA) on beam 8.3.1 using a Dectris Pilatus3 S 6M detector. Data were collected at $100 \mathrm{~K}$. Data indexing, integration and scaling were carried out using XDS. ${ }^{36}$ Anisotropic scaling was used for one of the datasets (PDB ID 6OUG). ${ }^{37}$ Both structures were solved by molecular replacement using Phaser ${ }^{38}$ in the CCP4 suite, ${ }^{39}$ with a single tetramer (PDB ID 6BMZ) as a search model. ${ }^{34}$ Refinement was carried out in Phenix ${ }^{40}$ with model building in Coot. ${ }^{41}$ Polder maps were calculated using Phenix. ${ }^{42}$ Pore diameters were calculated using CHUNNEL. ${ }^{43}$

\section{Molecular dynamics simulations.}

The X-ray crystal structure of M2(22-46) WT in complex with spiro-adamantylamine molecule (PDB ID 6BMZ) ${ }^{34}$ was used as the starting structure after mutating Val27 to Ala. The M2(22-46) V27A - spiro-adamantylamine complex was embedded in a hydrated POPC lipid bilayer and the system was subjected to all-atom MD simulations using Desmond. $34,44,45$ The POPC lipid bilayer extended $30 \AA$ beyond the protein, resulting in a system including ca 200 lipid molecules to allow the formation of two layers without interdigitation (Fig. S3). Membrane creation and system solvation were conducted with the "System Builder" utility of Desmond (Schrodinger, Cambridge, MA). ${ }^{44,45}$ Protein-ligand interactions were modelled using OPLS $2005 .{ }^{46-48}$ The TIP3 $\mathrm{P}^{49}$ model was used for water. This setup resulted in a simulation box of $88 \times 88 \times 105 \AA^{3}$ dimensions and ca 19000 water molecules. Calculation of long-range electrostatic interactions was carried out using the particle mesh Ewald method. ${ }^{50,51}$ A constant temperature was maintaned in all simulations using NoséHoover thermostat, and the Martyna-Tobias-Klein method was employed to control the pressure. ${ }^{52}$ Multistep RESPA integrator ${ }^{53}$ was used to integrate the equations of motion with an inner time step of $2 \mathrm{fs}$ for bonded interactions and non-bonded interactions within a cutoff of $9 \AA$. An outer time step of 6.0 fs was used for non-bonded interactions beyond the cutoff. The MD simulation protocol is included in the Supporting Information. A $300 \mathrm{~ns}$ NPT simulation without restraints was performed. Within this time, the RMSD of $\mathrm{C}_{\mathrm{a}}$ carbons of the protein reached a plateau, and the systems were considered to be equilibrated. For structural analyses, snapshots of the different systems were created with $\mathrm{VMD}^{54}$ or Maestro. 55 Trajectories were analyzed with Maestro, Gromacs, ${ }^{56,57}$ and VMD. Measurements were carried out with Gromacs tools. For the calculation of hydrogen bonds, a cut-off angle of $30^{\circ}$ of deviation from $180^{\circ}$ between the donor-hydrogen-acceptor atoms and a cut-off distance of $3.5 \AA$ A between the donor and acceptor atoms were applied.

\section{RESULTS AND DISCUSSION}

\section{X-ray crystal structures of spiro-adamantyl amine bound to M2(22-46) V27A and M2(21-61) V27A.}

We have used LCP crystallization techniques ${ }^{29}$ to solve two X-ray crystal structures of the adamantane-resistant M2 V27A mutant: one $2.5 \AA$ structure of M2(22-46) V27A in complex with spiro-adamantyl amine (PDB ID 6NV1), and one $3.0 \AA$ Astructure of M2(2161) V27A in complex with spiro-adamantyl amine (PDB ID 6OUG). The binding site of the spiro-adamantyl amine inhibitor is nearly identical in the two structures (Fig. S2a). Cocrystallization with a maltose neopentyl glycol (MNG) detergent additive appears to have stabilized M2 in its tetrameric form; in the absence of this detergent, the protein crystallized 
as an antiparallel dimer. The asymmetric unit of each structure contains two tetramers of M2

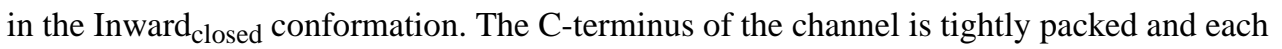
monomer helix has a kink of $13^{\circ}$ at residue Gly34. In the structure of the M2(22-46) V27A construct (6NV1), we observe a total of eight ordered monoolein molecules in the asymmetric unit interacting with the hydrophobic face of each monomer helix.

Consistent with previous X-ray crystal structures of the WT and S31N mutant M2 channel, here we observe ordered water molecules above the channel's gating His37 residues (Fig. S2b,c). ${ }^{27,28,34,58,59}$ In the $2.5 \AA$ resolution structure of M2(22-46) V27A (6NV1), four ordered water molecules are within hydrogen bonding distance of Ala30 carbonyls ("Ala30 layer") and spiro-adamantyl amine, and four waters are observed forming hydrogen bonds to Gly34 carbonyls ("Gly34 layer"). The Gly34 layer waters also form hydrogen bonds with the channel's gating His37 residues (Fig. 1). In the 3.0 A resolution structure of M2(21-61) (6OUG), we only observe electron density corresponding to two waters in the Gly34 layer. This is likely a result of increased molecular motion within the channel pore in this lower resolution structure. In the following discussion of adamantane resistance in the V27A mutant and the spiro-adamantyl amine binding mechanism, we will focus on the structure of the M2(22-46) V27A construct (6NV1) because it was solved to higher resolution and shows the contents of the channel pore in greater detail.

\section{Mechanism of amantadine and rimantadine resistance in the V27A mutant.}

The Val27 to Ala mutation at the N-terminus of M2 widens the channel pore at the adamantane binding site. In the M2 WT channel, four symmetry related Val27 residues form a valve that completely occludes the $\mathrm{N}$-terminus of the channel in the Inward $_{\text {closed }}$ conformation. ${ }^{34,58}$ Breathing motions of this Val27 gate are predicted to allow water, ions, and drugs/inhibitors to enter the N-terminus of the WT channel. ${ }^{60,61}$ The adamantyl group of the bound adamantane drugs interacts with the hydrophobic surface formed by the Val27 residues. ${ }^{34,59}$ When residue 27 is mutated from Val to Ala, these hydrophobic contacts are removed and the adamantanes (amantadine and rimantadine) can no longer inhibit the channel. In the crystal structure of M2(22-46) V27A bound to spiro-adamantyl amine, we observe that the width of the channel pore is $3.0 \AA$ at residue Ala27. For comparison, in the structure of M2(22-46) WT bound to amantadine (6BKK), the diameter at Val27 is $0.7 \AA{ }^{43}$ This widening of the $\mathrm{N}$-terminal channel pore is consistent with a previous solution NMR model of M2(18-60) V27A in DHPC micelles. ${ }^{62}$ The V27A mutant M2 channel conducts protons at an increased rate relative to the WT channel. ${ }^{8,62}$ This could be explained by the widened $\mathrm{N}$-terminal pore, as water and hydronium ions would have greater access to the region of the pore above the His37 gate. Though rimantadine is slightly longer than amantadine, the length of the compound is not sufficient to allow for the drug ammonium group to form hydrogen bonds with Ala30 layer waters while also allowing the adamantyl group to engage in hydrophobic contacts with the N-terminal portion of the pore near residue 27, and so rimantadine is not able to inhibit the V27A channel (Fig. S4). 


\section{Spiro-adamantyl amine shifts its binding position depending on which residue is present at position 27.}

Though mutation of Val27 to Ala prevents amantadine and rimantadine from inhibiting the channel, longer and bulkier inhibitors such as spiro-adamantyl amine can bind to the wider $\mathrm{N}$-terminal pore of V27A mutant and inhibit its proton conduction (Fig. 1). In the structure of the spiro-adamantyl amine complex with V27A (6NV1), we observe that the ammonium group of the spiro-adamantyl amine inhibitor localizes to approximately the same position as the ammonium group of amantadine in complex with M2(22-46) WT (6BKK) from our previous work. ${ }^{34}$ When the two structures are aligned, the position of the ammonium groups are nearly identical $(0.9 \AA)$ when comparing the WT-amantadine vs. V27A-spiro-adamantyl amine complexes. In both structures there are ordered waters in the Ala30 and Gly34 solvent layers, and the ammonium group of the bound drug/inhibitor forms hydrogen bonds with two waters in the Ala30 layer. The hydrogen bonds formed between the inhibitor nitrogen and pore waters are short (2.4-2.6 $\AA$ ), which supports the ionization state we have chosen for the ligand. The binding of the spiro-adamantyl amine inhibitor to the top of this hydrogen-bonded water network blocks protons from being transported down through these water wires to the gating His37 residue. The tautomer we have assigned for the His37 residues (Nع2-protonated $\tau$-tautomer) is as previously observed in ssNMR experiments. ${ }^{63}$

In our previous work, ${ }^{34}$ we characterized the binding of this same spiro-adamantyl amine inhibitor to the M2(22-46) WT channel (6BMZ). When spiro-adamantyl amine binds to the WT channel, the inhibitor's adamantyl group occupies approximately the same position in the pore as the adamantyl group of bound amantadine or rimantadine in M2 WT. However, the longer hydrophobic scaffold of the inhibitor completely displaces the four waters in the Ala30 layer (Fig. 1), such that its ammonium group binds deeper in the channel pore and forms hydrogen bonds with waters in the Gly34 solvent layer. Thus, the spiro-adamantyl amine inhibitor blocks proton conductance in both the WT and the adamantane-resistant V27A M2 channel by shifting its binding position depending on which residue is present at position 27 . This dual inhibitor exploits the M2 channel's functionally essential ability to stabilize positive charges at multiple sites within the channel pore. ${ }^{26}$

\section{Crystal structure of the M2(21-61) V27A construct in complex with spiro-adamantyl amine.}

The TM domain of $\mathrm{M} 2$ is the minimum construct needed for selective, unidirectional proton transport. ${ }^{6,7}$ However, truncation of the C-terminus and cytosolic helix (residues 46-60) has minor effects on the proton conduction properties relative to the full length channel. ${ }^{64}$ More importantly, the cyto helix also has functional importance for budding and membrane scission..$^{9,10,65}$ Constructs including the cytosolic helix were more difficult to crystallize relative to the TM construct, presumably because of the conformational mobility of this domain. Nevertheless, after much optimization, we were able to grow crystals of M2(21-61) V27A in complex with spiro-adamantyl amine and solve a structure to $3.0 \AA$ A resolution (6OUG).

In structure 6OUG, we observe electron density for residues 22-56. The structural features of the TM domain (22-46) are similar for both the M2(22-46) and the M2(21-61) constructs, with Ca RMSD $=0.25 \AA$. The binding site of the spiro-adamantyl amine 
inhibitor is nearly identical, although in structure 6OUG there are fewer ordered waters visible in the channel pore between the inhibitor ammonium group and the gating His 37 residues (likely a result of the lower resolution of this structure). Residues 47-56 form an ahelix that extends straight down from the TM helix. We observe tighter packing of the tetrameric bundle at the $\mathrm{C}$-terminus relative to structures that include only the TM domain (Fig. 2b,c). At residue Arg45, the minimum pore diameter is $2.8 \AA$ in the structure of M2(22-46) V27A and $1.0 \AA$ in the structure of M2(21-61) V27A. ${ }^{43}$ All four Asp44 and Arg45 residues in the structure of M2(21-61) V27A face the aqueous pore. Residue Phe48 faces the channel pore and is well-positioned for the stabilization of the positive charge on Arg45 through cation- $\pi$ interactions. In the native M2 protein, Cys50 is palmitoylated. ${ }^{66}$ Here, Cys50 has been mutated to Ser to facilitate peptide synthesis and purification and to prevent oligomerization through the formation of disulfide bonds. We observe that the Ser50 side chain faces away from the aqueous pore and toward the lipid bilayer.

Several NMR studies predict that the cytosolic helix of M2 has a-helical secondary structure, with either a turn or a disordered region connecting the cyto helix to the TM domain. In these NMR structures, the TM helix ends and the turn / disordered region begins at either residue Leu46 (2KWX, 2RLF, 2KIH) ${ }^{62,67,68}$ or residue Phe48 (2L0J, 2N70). ${ }^{69,70}$

The conformation of the channel's C-terminus that we observe in the X-ray crystal structure here differs from solution and solid state NMR structures. Solution NMR studies have shown that the conformation of the juxta-membrane domain connecting the TM and cytosolic helices is sensitive to solubilizing environments. ${ }^{71}$ Thus, the conformation of the monomer helices from residue 47 to 56 is possibly influenced by crystal contacts at the channel's C-terminus or choice of lipid mimetic. We include structure 6OUG here as a comparison to the shorter M2(22-46) V27A construct and also because structural information about the helical turn immediately below residue 46 may prove useful for understanding proton conduction through the $\mathrm{C}$-terminus of the channel.

\section{Molecular dynamics simulations of M2(22-46) V27A in complex with spiro-adamantyl amine.}

The contents of the M2 channel pore differ in the two crystal structures described here; fewer waters are observed in the structure of M2(21-61) V27A in complex with spiroadamantyl amine. We carried out molecular dynamics simulations to examine the ordering of water within the pore at room temperature. M2(22-46) was simulated in the Inward closed $_{\text {d }}$ conformation at $\mathrm{pH} 8.0$ with a neutral form assigned for all four His 37 residues and a protonated ammonium group for the ligand. The starting structure was produced after applying the V27A mutation to the structure of spiro-adamantyl amine in complex with

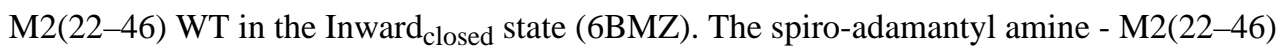
V27A protein complex was subjected to MD simulations in a POPC bilayer for $300 \mathrm{~ns}$. The simulated M2(22-46) V27A - ligand complex was stable, and in all cases the M2(22-46) V27A tetramer showed no large conformational changes in the course of the simulations, as demonstrated by RMSDs smaller than $1.5 \AA$ for M2 (22-46) $\mathrm{C}_{\mathrm{a}}$-carbons with respect to the starting structure (Table S2, Fig. S5). Also, the individual chains of the tetramer did not deviate from the starting structure, with RMSDs $\leq 1.1 \AA$ A for M2 (27-46) Ca carbons and no changes observed in selected torsion angles of each chain (Tables S3,S4). 
In agreement with the crystal structure, the spiro-adamantyl amine moved towards the $\mathrm{N}$ terminus by ca. $2 \AA$ within the first $50 \mathrm{~ns}$ of the simulation. The average angle between the central axis of the pore and the adamantane symmetry axis was ca $8^{\circ}$ (Table S2), i.e., the ammonium group of the spiro-adamantylamine compound oriented towards the $\mathrm{C}$-terminus, and the adamantyl cage localized between Ala27 and Ser31. The ligand's ammonium group forms hydrogen bonds with water molecules between the ligand and the His 37 residues. Although the simulation was initiated with only 4 water molecules within the pore, water rushes in within $85 \mathrm{nsec}$, forming two layers that are stabilized that remain stable for the remaining $215 \mathrm{nsec}$ of the simulation. These two layers of waters can be seen in the radial distribution density plotted for the distance between the spiro-adamantyl amine ammonium group and the oxygen atoms from water (Fig. S6). The top layer consists of four water molecules that form hydrogen bonds with the ligand's ammonium group and the Ala30 carbonyls. The lower layer of four water molecules is stabilized by hydrogen-bonding interactions involving the carbonyls of Gly34 as well as the His37 side chain. $\mathrm{Cl}^{-}$ions were present in the simulation to reduce the electrostatic potential created by positively charged groups. $\mathrm{No} \mathrm{Cl}^{-}$ions entered the pore, as indicated by the average Cl-N distance (Table S2). Previous works ${ }^{72,73,74}$ mention the presence of a $\mathrm{Cl}$ - ion close to His 37 because the His 37 residues were charged. In our experimental structure and MD simulations, a $\mathrm{Cl}$ - anion was found in the vicinity of Trp41. This is not unexpected because the spiro-adamantyl ligand is in the ammonium form. The spiro-adamantyl amine - M2(22-46) V27A complex in the Xray structure was perfectly superimposed with the structure from the $300 \mathrm{~ns}$ MD simulation, with the RMSD of the ligand being less than $1 \AA$ (Fig. 3).

We have analyzed the trajectories of the MD simulation to determine whether the waters in the channel pore remain tightly bound or if they are exchanged during the simulation. A total of 31 water molecules are found to transiently occupy the Ala30 and Gly34 water layers (Fig. S7 i.e. from the inhibitor ammonium to His37, which is a distance of $7 \AA$ ), with residence times on the order of $50-100 \mathrm{nsec}$. Out of the 31 total waters, 13 diffuse rapidly in and out of this region of the pore. The remaining 18 water molecules in Figure S8 are remain inside the binding cavity with a mean residence time of approximately $40 \mathrm{~ns}$. Interestingly, these waters are not fixed, but instead dynamically transition between the top and bottom water layers with an average correlation time of $2 \mathrm{nsec}$. Thus, the exchange of waters within the channel is much more rapid than the exchange in and out of the channel.

\section{CONCLUSIONS}

These X-ray crystal structures reveal the mechanism of adamantane resistance in the V27A channel. Hydrophobic contacts critical for drug binding are removed when Val27 is mutated to Ala. We also observe that the spiro-adamantyl amine inhibitor blocks proton conduction in both the WT and V27A mutant M2 channels by shifting its binding site depending on whether Val or Ala are present at position 27, and that a network of either one or two layers of hydrogen-bonded waters is formed. MD simulations in POPC hydrated bilayers accurately predict the X-ray M2 V27A structure (6NV1).

These findings are consistent with MD simulations in which positive charges within the M2 pore are predicted to be stabilized at multiple positions within the channel. ${ }^{26}$ The structural 
characterization of this dual inhibition mechanism is intriguing from the perspective of molecular recognition and drug design. Though there are a limited number of M2 mutants that retain the electrophysiological properties necessary for viral replication, circulating populations of the influenza virus often contain a mix of M2 sequences. The design of drugs to target more than one sequence could be a successful strategy for targets such as M2. Structural information about adamantane-resistant mutants can guide this process.

\section{ACCESSION CODES}

PDB ID 6NV1, PDB ID 6OUG.

\section{Supplementary Material}

Refer to Web version on PubMed Central for supplementary material.

\section{ACKNOWLEDGMENT}

J.L.T. and W.F.D. were supported by NIH grants R35-GM122603 and R01-GM117593. J.W. was supported by NIH grant R33-AI119187. J.T and M.C. were supported by Science Foundation Ireland grants 12/IA/1255 and 16/IA/ 4435. A.K. is grateful to Chiesi Hellas for supporting the Ph.D work of Ath.K. Use of the LCP crystallization robot was made possible by National Center for Research Resources Grant 1S10RR027234-01. The authors thank Pil Seok Chae (Hanyang University, Seoul, South Korea) for providing MNG detergent for crystallization trials. J.T. and M.C. thank David Aragão and Valerie Pye for help with diffraction measurements and structure determination using first ever crystals of the M2-V27A TM domain. Data collection was carried out at ALS 8.3.1. Beamline 8.3.1 at the Advanced Light Source is operated by the University of California Office of the President, Multicampus Research Programs and Initiatives grant MR-15-328599 and NIGMS grants P30 GM124169 and R01 GM124149. The authors thank George Meigs and James Holton at ALS 8.3.1 for support during data collection.

Funding Sources

No competing financial interests have been declared.

This research was funded by NIH grants R35-GM122603, R01-GM117593, R33-AI119187, P30-GM124169, and R01-GM124149; SFI grants 12/IA/1255 and 16/IA/4435; NCRR grant 1S10RR027234-01; and University of California Office of the President grant MR-15-328599.

\section{ABBREVIATIONS}

$\begin{array}{ll}\text { C }_{\mathbf{a}} & \text { alpha carbon } \\ \text { HPLC } & \text { high pressure liquid chromatography } \\ \text { LCP } & \text { lipid cubic phase } \\ \text { M1 } & \text { matrix 1 protein } \\ \text { M2 } & \text { matrix 2 protein } \\ \text { MALDI-MS } & \text { matrix-assisted laser desorption/ionization mass spectrometry } \\ \text { MD } & \text { molecular dynamics } \\ \text { MNG } & \text { maltose neopentyl glycol } \\ \text { NMR } & \text { nuclear magnetic resonance }\end{array}$




$\begin{array}{ll}\text { POPC } & \text { phosphatidylcholine } \\ \text { S31N } & \text { serine } 31 \text { to asparagine } \\ \text { RMSD } & \text { root-mean-square deviation } \\ \text { TEVC } & \text { two electrode voltage clamp } \\ \text { TM } & \text { transmembrane } \\ \text { V27A } & \text { valine } 27 \text { to alanine } \\ \text { WT } & \text { wild type }\end{array}$

\section{REFERENCES}

(1). Park EK; Castrucci MR; Portner A; Kawaoka Y (1998) The M2 ectodomain is important for its incorporation into influenza A virions. J. Virol 72 (3), 2449-2455. [PubMed: 9499106]

(2). Chizhmakov IV; Geraghty FM; Ogden DC; Hayhurst A; Antoniou M; Hay AJ (1996) Selective proton permeability and $\mathrm{pH}$ regulation of the influenza virus $\mathrm{M} 2$ channel expressed in mouse erythroleukaemia cells. J. Physiol 494 (2), 329-336. [PubMed: 8841994]

(3). Mould JA; Drury JE; Frings SM; Kaupp UB; Pekosz A; Lamb RA; Pinto LH (2000) Permeation and activation of the M-2 ion channel of influenza A virus. J. Biol. Chem 275 (40), 3103831050. [PubMed: 10913133]

(4). Martin K; Helenius A (1991) Nuclear transport of influenza virus ribonucleoproteins: The viral matrix protein (M1) promotes export and inhibits import. Cell 67 (1), 117-130. [PubMed: 1913813]

(5). Sakaguchi T,LG, Lamb RA (1996) The ion channel activity of the influenza virus M2 protein affects transport through the Golgi apparatus. J. Cell Biol 133 (4), 733-747. [PubMed: 8666660]

(6). Duff KC; Ashley RH (1992) The transmembrane domain of influenza A M2 protein forms amantadine-sensitive proton channels in planar lipid bilayers. Virology 190 (1), 485-489. [PubMed: 1382343]

(7). Ma CL; Polishchuk AL; Ohigashi Y; Stouffer AL; Schon A; Magavern E; Jing XH; Lear JD; Freire E; Lamb RA; DeGrado WF; Pinto LH (2009) Identification of the functional core of the influenza A virus A/M2 proton-selective ion channel. Proc. Natl. Acad. Sci. U. S. A 106 (30), 12283-12288. [PubMed: 19590009]

(8). Balannik V; Carnevale V; Fiorin G; Levine BG; Lamb RA; Klein ML; DeGrado WF; Pinto LH (2010) Functional studies and modeling of pore-lining residue mutants of the influenza A virus M2 ion channel. Biochemistry 49 (4), 696-708. [PubMed: 20028125]

(9). Rossman JS; Jing X; Leser GP; Lamb RA (2010) Influenza virus M2 protein mediates ESCRTindependent membrane scission. Cell 142 (6), 902-913. [PubMed: 20850012]

(10). Schmidt NW; Mishra A; Wang J; DeGrado WF; Wong GCL (2013) Influenza virus A M2 protein generates negative gaussian membrane curvature necessary for budding and scission. J. Am. Chem. Soc 135 (37), 13710-13719. [PubMed: 23962302]

(11). McCown MF; Pekosz A (2006) Distinct domains of the influenza A virus M2 protein cytoplasmic tail mediate binding to the M1 protein and facilitate infectious virus production. Journal of Virology 80 (16), 8178-8189. [PubMed: 16873274]

(12). Sheu TG; Fry AM; Garten RJ; Deyde VM; Shwe T; Bullion L; Peebles PJ; Li Y; Klimov AI; Gubareva LV (2011) Dual Resistance to Adamantanes and Oseltamivir Among Seasonal Influenza A(H1N1) Viruses: 2008-2010. J. Infect. Dis 203 (1), 13-17. [PubMed: 21148491]

(13). Zhou J; Zou L; Zhang X; Liao J; Ni H; Hou N; Wang Y; Li H; Wu J; Jonges M; Meijer A; Koopmans M; Ke C (2011) Adamantane- and oseltamivir-resistant seasonal A (H1N1) and pandemic A (H1N1) 2009 influenza viruses in Guangdong, China, during 2008 and 2009. J. Clin. Microbiol 49 (7), 2651-2655. [PubMed: 21593267] 
(14). Garcia V; Aris-Brosou S (2014) Comparative dynamics and distribution of influenza drug resistance acquisition to protein $\mathrm{m} 2$ and neuraminidase inhibitors. Mol. Biol. Evol 31 (2), 355363. [PubMed: 24214415]

(15). Dong G; Peng C; Luo J; Wang C; Han L; Wu B; Ji G; He H (2015) Adamantane-resistant influenza A viruses in the world (1902-2013): frequency and distribution of M2 gene mutations. Plos One 10 (3), e0119115. [PubMed: 25768797]

(16). Furuse Y; Suzuki A; Oshitani H (2009) Large-Scale Sequence Analysis of M Gene of Influenza A Viruses from Different Species: Mechanisms for Emergence and Spread of Amantadine Resistance. Antimicrob. Agents Chemother 53 (10), 4457-4463. [PubMed: 19651904]

(17). Baranovich T; Bahl J; Marathe BM; Culhane M; Stigger-Rosser E; Darnell D; Kaplan BS; Lowe JF; Webby RJ; Govorkova EA (2015) Influenza A viruses of swine circulating in the United States during 2009-2014 are susceptible to neuraminidase inhibitors but show lineage-dependent resistance to adamantanes. Antivir. Res 117, 10-19. [PubMed: 25701593]

(18). Durrant MG; Eggett DL; Busath DD (2015) Investigation of a recent rise of dual amantadineresistance mutations in the influenza A M2 sequence. BMC Genetics 16 (Suppl 2), S3. [PubMed: 25953496]

(19). Moasser E; Moasser A; Zaraket H (2019) Incidence of antiviral drug resistance markers among human influenza A viruses in the Eastern Mediterranean Region, 2005-2016. Infect. Genet. Evol 67, 60-66. [PubMed: 30389548]

(20). Suzuki H; Saito R; Masuda H; Oshitani H; Sato M; Sato I (2003) Emergence of amantadineresistant influenza A viruses: epidemiological study. J. Infect. Chemother 9 (3), 195-200. [PubMed: 14513385]

(21). Balannik V; Wang J; Ohigashi Y; Jing X; Magavern E; Lamb RA; Degrado WF; Pinto LH (2009) Design and pharmacological characterization of inhibitors of amantadine-resistant mutants of the M2 ion channel of influenza A virus. Biochemistry 48 (50), 11872-11882. [PubMed: 19905033]

(22). Hu Y; Musharrafieh R; Ma C; Zhang J; Smee DF; DeGrado WF; Wang J (2017) An M2-V27A channel blocker demonstrates potent in vitro and in vivo antiviral activities against amantadinesensitive and -resistant influenza A viruses. Antiviral Res. 140, 45-54. [PubMed: 28087313]

(23). Rey-Carrizo M; Barniol-Xicota M; Ma C; Frigolé-Vivas M; Torres E; Naesens L; Llabrés S; Juárez-Jiménez J; Luque FJ; DeGrado WF; Lamb RA; Pinto LH; Vázquez S (2014) Easily accessible polycyclic amines that inhibit the wild-type and amantadine-resistant mutants of the M2 channel of influenza A virus. J. Med. Chem 57 (13), 5738-5747. [PubMed: 24941437]

(24). Rey-Carrizo M; Gazzarrini S; Llabrés S; Frigolé-Vivas M; Juárez-Jiménez J; Font-Bardia M; Naesens L; Moroni A; Luque FJ; Vázquez S (2015) New polycyclic dual inhibitors of the wild type and the V27A mutant M2 channel of the influenza A virus with unexpected binding mode. Eur. J. Med. Chem 96, 318-329. [PubMed: 25899336]

(25). Rey-Carrizo M; Torres E; Ma C; Barniol-Xicota M; Wang J; Wu Y; Naesens L; DeGrado WF; Lamb RA; Pinto LH; Vázquez S (2013) 3-Azatetracyclo[5.2.1.1(5,8).0(1,5)]undecane derivatives: from wild-type inhibitors of the M2 ion channel of influenza A virus to derivatives with potent activity against the V27A mutant. J. Med. Chem 56 (22), 9265-9274. [PubMed: 24237039]

(26). Wang J; Ma CL; Fiorin G; Carnevale V; Wang T; Hu FH; Lamb RA; Pinto LH; Hong M; Kein ML; DeGrado WF (2011) Molecular dynamics simulation directed rational design of inhibitors targeting drug-resistant mutants of influenza A virus M2. J. Am. Chem. Soc 133 (32), 1283412841. [PubMed: 21744829]

(27). Thomaston JL; Alfonso-Prieto M; Woldeyes RA; Fraser JS; Klein ML; Fiorin G; DeGrado WF (2015) High-resolution structures of the M2 channel from influenza A virus reveal dynamic pathways for proton stabilization and transduction. Proc. Natl. Acad. Sci. U. S. A 112 (46), 14260-14265. [PubMed: 26578770]

(28). Thomaston JL; DeGrado WF (2016) Crystal structure of the drug-resistant S31N influenza M2 proton channel. Protein Sci. 25 (8), 1551-1554. [PubMed: 27082171]

(29). Caffrey M; Cherezov V (2009) Crystallizing membrane proteins using lipidic mesophases. Nat. Protoc 4 (5), 706-731. [PubMed: 19390528] 
(30). Höfer N; Aragão D; Caffrey M (2010) Crystallizing transmembrane peptides in lipidic mesophases. Biophys. J 99 (3), L23-L25. [PubMed: 20682243]

(31). Liu W; Caffrey M (2005) Gramicidin structure and disposition in highly curved membranes. J. Struct. Biol 150 (1), 23-40. [PubMed: 15797727]

(32). Chae PS; Rasmussen SGF; Rana R; Gotfryd K; Chandra R; Goren MA; Kruse AC; Nurva S; Loland CJ; Pierre Y; Drew D; Popot J-L; Picot D; Fox BG; Guan L; Gether U; Byrne B; Kobilka B; Gellman SH (2010) Maltose-neopentyl glycol (MNG) amphiphiles for solubilization, stabilization and crystallization of membrane proteins. Nat. Methods 7 (12), 1003-1008. [PubMed: 21037590]

(33). Cho KH; Husri M; Amin A; Gotfryd K; Lee HJ; Go J; Kim JW; Loland CJ; Guan L; Byrne B; Chae PS (2015) Maltose Neopentyl Glycol-3 (MNG-3) Analogues for Membrane Protein Study. Analyst 140 (9), 3157-3163. [PubMed: 25813698]

(34). Thomaston JLP,NF; Konstantinidi A; Wang J; Kolocouris A; DeGrado WF (2018) Inhibitors of the M2 proton channel engage and disrupt transmembrane networks of hydrogen-bonded waters. J. Am. Chem. Soc 140 (45), 15219-15226. [PubMed: 30165017]

(35). Thomaston JLW,Y; Polizzi N; Liu L; Wang J; DeGrado WF (2019) X-ray crystal structure of the influenza A M2 proton channel S31N mutant in two conformational states: an open and shut case. J. Am. Chem. Soc 141 (29), 11481-11488. [PubMed: 31184871]

(36). Kabsch W (2010) XDS. Acta Crystallogr., Sect. D: Biol. Crystallogr 66 (2), 125-132. [PubMed: 20124692]

(37). Strong M; Sawaya MR; Wang S; Phillips M; Cascio D; Eisenberg D (2006) Toward the structural genomics of complexes: Crystal structure of a PE/PPE protein complex from Mycobacterium tuberculosis. Proc. Natl. Acad. Sci. U. S. A 103 (21), 8060-8065. [PubMed: 16690741]

(38). McCoy AJ; Grosse-Kunstleve RW; Adams PD; Winn MD; Storoni LC; Read RJ (2007) Phaser crystallographic software. J. Appl. Crystallogr 40, 658-674. [PubMed: 19461840]

(39). Winn MD; Ballard CC; Cowtan KD; Dodson EJ; Emsley P; Evans PR; Keegan RM; Krissinel EB; Leslie AGW; McCoy A; McNicholas SJ; Murshudov GN; Pannu NS; Potterton EA; Powell HR; Read RJ; Vagin A; Wilson KS (2011) Overview of the CCP4 suite and current developments. Acta Crystallogr., Sect. D: Biol. Crystallogr 67, 235-242. [PubMed: 21460441]

(40). Adams PD; Afonine PV; Bunkoczi G; Chen VB; Davis IW; Echols N; Headd JJ; Hung LW; Kapral GJ; Grosse-Kunstleve RW; McCoy AJ; Moriarty NW; Oeffner R; Read RJ; Richardson DC; Richardson JS; Terwilliger TC; Zwart PH (2010) PHENIX: a comprehensive Python-based system for macromolecular structure solution. Acta Crystallogr., Sect. D: Biol. Crystallogr 66, 213-221. [PubMed: 20124702]

(41). Emsley P; Lohkamp B; Scott WG; Cowtan K (2010) Features and development of Coot. Acta Crystallogr., Sect. D: Biol. Crystallogr 66, 486-501. [PubMed: 20383002]

(42). Liebschner D; Afonine PV; Moriarty NW; Poon BK; Sobolev OV; Terwilliger TC; Adams PD (2017) Polder maps: improving OMIT maps by excluding bulk solvent. Acta Crystallogr., Sect. D: Biol. Crystallogr 73 (Pt 2), 148-157.

(43). Coleman RG; Sharp KA (2009) Finding and Characterizing Tunnels in Macromolecules with Application to Ion Channels and Pores. Biophys. J 96 (2), 632-645. [PubMed: 18849407]

(44). Bowers KJ; Chow E; Xu H; Dror RO; Eastwood MP; Gregersen BA; Klepeis JL; Kolossvary I; Moraes MA; Sacerdoti FD; Salmon JK; Shan Y; Shaw DE (2006) Scalable algorithms for molecular dynamics simulations on commodity clusters. Proceedings of the 2006 ACM/IEEE Conference on Supercomputing, ACM: Tampa, Florida, 43-43.

(45). Schrodinger L, (2012) Maestro-Desmond Interoperability Tools, version 3.1

(46). Jorgensen WL; Maxwell DS; Tirado-Rives J (1996) Development and Testing of the OPLS AllAtom Force Field on Conformational Energetics and Properties of Organic Liquids. J. Am. Chem. Soc 118 (45), 11225-11236.

(47). Kaminski GA; Friesner RA; Tirado-Rives J; Jorgensen WL (2001) Evaluation and Reparametrization of the OPLS-AA Force Field for Proteins via Comparison with Accurate Quantum Chemical Calculations on Peptides. J. Phys. Chem. B 105 (28), 6474-6487.

(48). Rizzo RC; Jorgensen WL (1999) OPLS All-Atom Model for Amines: Resolution of the Amine Hydration Problem. J. Am. Chem. Soc 121 (20), 4827-4836. 
(49). Jorgensen WL; Chandrasekhar J; Madura JD; Impey RW; Klein ML (1983) Comparison of simple potential functions for simulating liquid water. J. Chem. Phys 79 (2), 926-935.

(50). Essmann U; Perera L; Berkowitz ML; Darden T; Lee H; Pedersen LG (1995) A smooth particle mesh Ewald method. J. Chem. Phys 103 (19), 8577-8593.

(51). Darden T; York D; Pedersen L (1993) Particle mesh Ewald: An N·log(N) method for Ewald sums in large systems. J. Chem. Phys 98 (12), 10089-10092.

(52). Martyna GJ; Tobias DJ; Klein ML (1994) Constant pressure molecular dynamics algorithms. J. Chem. Phys 101 (5), 4177-4189.

(53). Humphreys DD; Friesner RA; Berne BJ (1994) A Multiple-Time-Step Molecular Dynamics Algorithm for Macromolecules. J. Phys. Chem 98 (27), 6885-6892.

(54). Humphrey W; Dalke A; Schulten K (1996) VMD: Visual molecular dynamics. J. Mol. Graph 14 (1), 33-38. [PubMed: 8744570]

(55). Schrodinger L (2008) Maestro, version 8.5

(56). Berendsen HJC; van der Spoel D; van Drunen R (1995) GROMACS: A message-passing parallel molecular dynamics implementation. Comput. Phys. Commun 91 (1), 43-56.

(57). Hess B; Kutzner C; van der Spoel D; Lindahl E (2008) GROMACS 4: Algorithms for Highly Efficient, Load-Balanced, and Scalable Molecular Simulation. J. Chem. Theory Comput 4 (3), 435-447. [PubMed: 26620784]

(58). Acharya R; Carnevale V; Fiorin G; Levine BG; Polishchuk AL; Balannik V; Samish I; Lamb RA; Pinto LH; DeGrado WF; Klein ML (2010) Structure and mechanism of proton transport through the transmembrane tetrameric M2 protein bundle of the influenza A virus. Proc. Natl. Acad. Sci. U. S. A 107 (34), 15075-80. [PubMed: 20689043]

(59). Stouffer AL; Acharya R; Salom D; Levine AS; Di Costanzo L; Soto CS; Tereshko V; Nanda V; Stayrook S; DeGrado WF (2008) Structural basis for the function and inhibition of an influenza virus proton channel. Nature 451 (7178), 596-U13. [PubMed: 18235504]

(60). Khurana E; Dal Peraro M; DeVane R; Vemparala S; DeGrado WF; Klein ML (2009) Molecular dynamics calculations suggest a conduction mechanism for the M2 proton channel from influenza A virus. Proc. Natl. Acad. Sci. U. S. A 106 (4), 1069-1074. [PubMed: 19144924]

(61). Yi M; Cross TA; Zhou HX (2008) A secondary gate as a mechanism for inhibition of the M2 proton channel by amantadine. J. Phys. Chem. B 112 (27), 7977-7979. [PubMed: 18476738]

(62). Pielak RM; Chou JJ (2010) Solution NMR structure of the V27A drug resistant mutant of influenza A M2 channel. Biochem. Biophys. Res. Commun 401 (1), 58-63. [PubMed: 20833142]

(63). Hu FH; Luo WB; Hong M (2010) Mechanisms of proton conduction and gating in influenza M2 proton channels from solid-state NMR. Science 330, 505-508. [PubMed: 20966251]

(64). Tobler K; Kelly ML; Pinto LH; Lamb RA (1999) Effect of cytoplasmic tail truncations on the activity of the $\mathrm{M}(2)$ ion channel of influenza A virus. J. Virol 73 (12), 9695-9701. [PubMed: 10559278]

(65). Roberts KL; Leser GP; Ma C; Lamb RA (2013) The amphipathic helix of influenza A virus M2 protein is required for filamentous bud formation and scission of filamentous and spherical particles. J. Virol 87 (18), 9973-9982. [PubMed: 23843641]

(66). Sugrue RJ; Belshe RB; Hay AJ (1990) Palmitoylation of the influenza a virus M2 protein. Virology 179 (1), 51-56. [PubMed: 2219738]

(67). Pielak RM; Schnell JR; Chou JJ (2009) Mechanism of drug inhibition and drug resistance of influenza A M2 channel. Proc. Natl. Acad. Sci. U. S. A 106 (18), 7379-7384. [PubMed: 19383794]

(68). Schnell JR; Chou JJ (2008) Structure and mechanism of the M2 proton channel of influenza A virus. Nature 451 (7178), 591-595. [PubMed: 18235503]

(69). Andreas LB; Reese M; Eddy MT; Gelev V; Ni QZ; Miller EA; Emsley L; Pintacuda G; Chou JJ; Griffin RG (2015) Structure and Mechanism of the Influenza A M218-60 Dimer of Dimers. J. Am. Chem. Soc 137 (47), 14877-14886. [PubMed: 26218479]

(70). Sharma M; Yi M; Dong H; Qin H; Peterson E; Busath DD; Zhou HX; Cross TA (2010) Insight into the mechanism of the influenza a proton channel from a structure in a lipid bilayer. Science 330 (6003), 509-512. [PubMed: 20966252] 
(71). Claridge JK; Aittoniemi J; Cooper DM; Schnell JR (2013) Isotropic Bicelles Stabilize the Juxtamembrane Region of the Influenza M2 Protein for Solution NMR Studies. Biochemistry 52 (47), 8420-8429. [PubMed: 24168642]

(72). Llabrés S; Juárez-Jiménez J; Masetti M; Leiva R; Vázquez S; Gazzarrini S; Moroni A; Cavalli A; Javier Luque F (2016) Mechanism of the Pseudoirreversible Binding of Amantadine to the M2 Proton Channel. J. Am. Chem. Soc 138, 15345-15358. [PubMed: 27933932]

(73). Mustafa M; Henderson DJ; Busath DD (2009) Free-energy profiles for ions in the influenza M2TMD channel. Proteins 76, 794-807. [PubMed: 19296508]

(74). Wei C; Pohorille A (2013) Activation and proton transport mechanism in influenza A M2 channel. Biophys. J 105, 2036-2045. [PubMed: 24209848] 


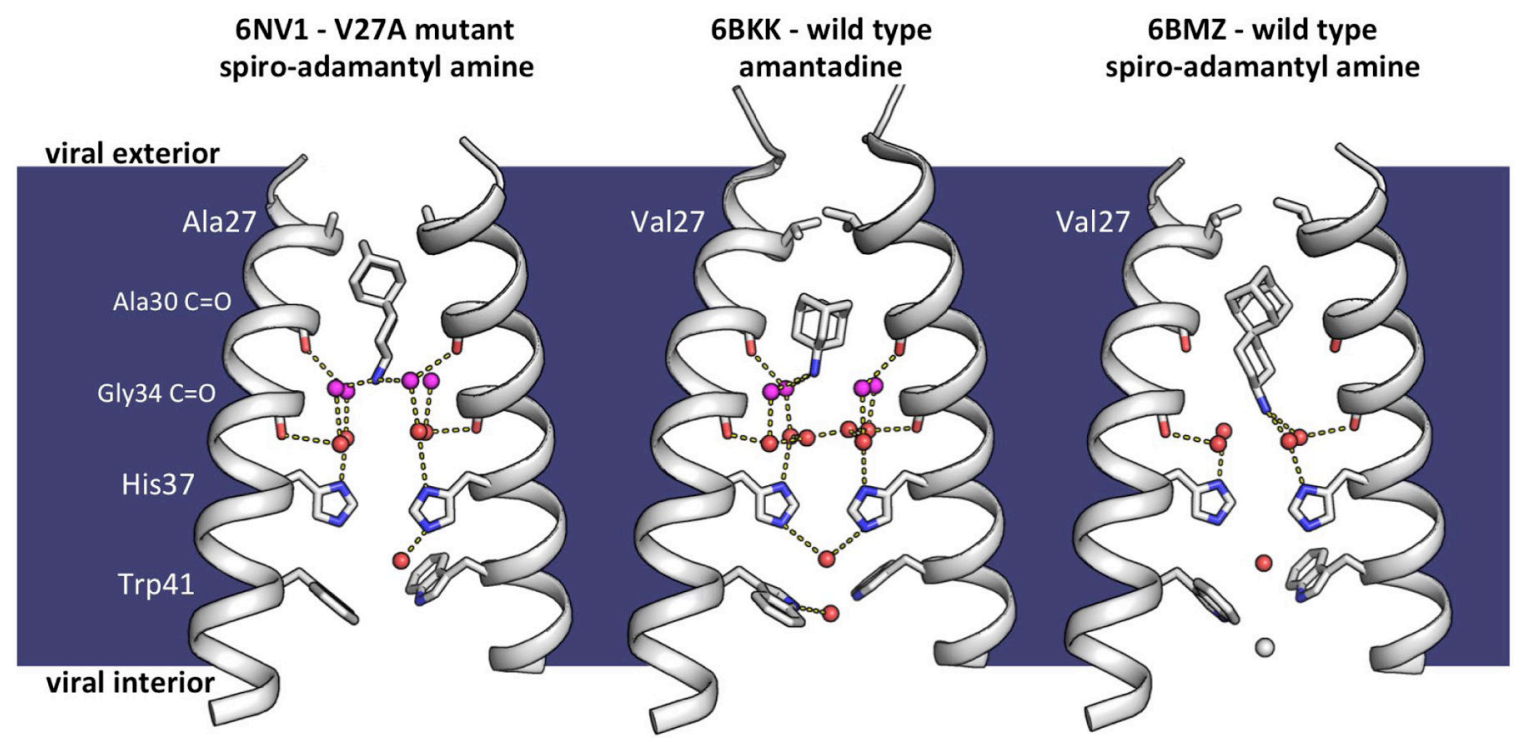

Figure 1.

Mechanism of adamantane drug resistance in the V27A mutant channel, and mechanism of dual inhibition by spiro-adamantyl amine. Front and back monomer helices have been removed to clearly show the contents of the channel pore. Pink spheres correspond to waters that form hydrogen bonds with the Ala30 carbonyls, and red spheres correspond to waters forming hydrogen bonds with Gly34 carbonyls. Left to right: Newly solved structure of M2(22-46) V27A bound to a spiro-adamantyl amine inhibitor (6NV1, monomer chains B and D), M2(22-46) WT bound to amantadine (6BKK, chains B and D), ${ }^{34}$ and M2(22-46) WT bound to spiro-adamantyl amine (6BMZ, chains B and D). ${ }^{34}$ When spiro-adamantyl amine binds to the V27A channel (6NV1), the ammonium group of the inhibitor localizes to the same position as the ammonium of amantadine in the WT structure (6BKK). The adamantyl group is positioned higher in the channel pore, occupying the extra space created by the Val27 to Ala mutation. In the previously solved structure of spiro-adamantyl amine inhibitor bound to the WT channel (6BMZ), the adamantyl group of the inhibitor binds lower in the channel pore, and the four waters in the Ala30 layer are displaced. For the spiroadamantyl amine bound structures, a network of one or two layers of hydrogen-bonded waters is formed depending whether Val or Ala is present at position 27. 


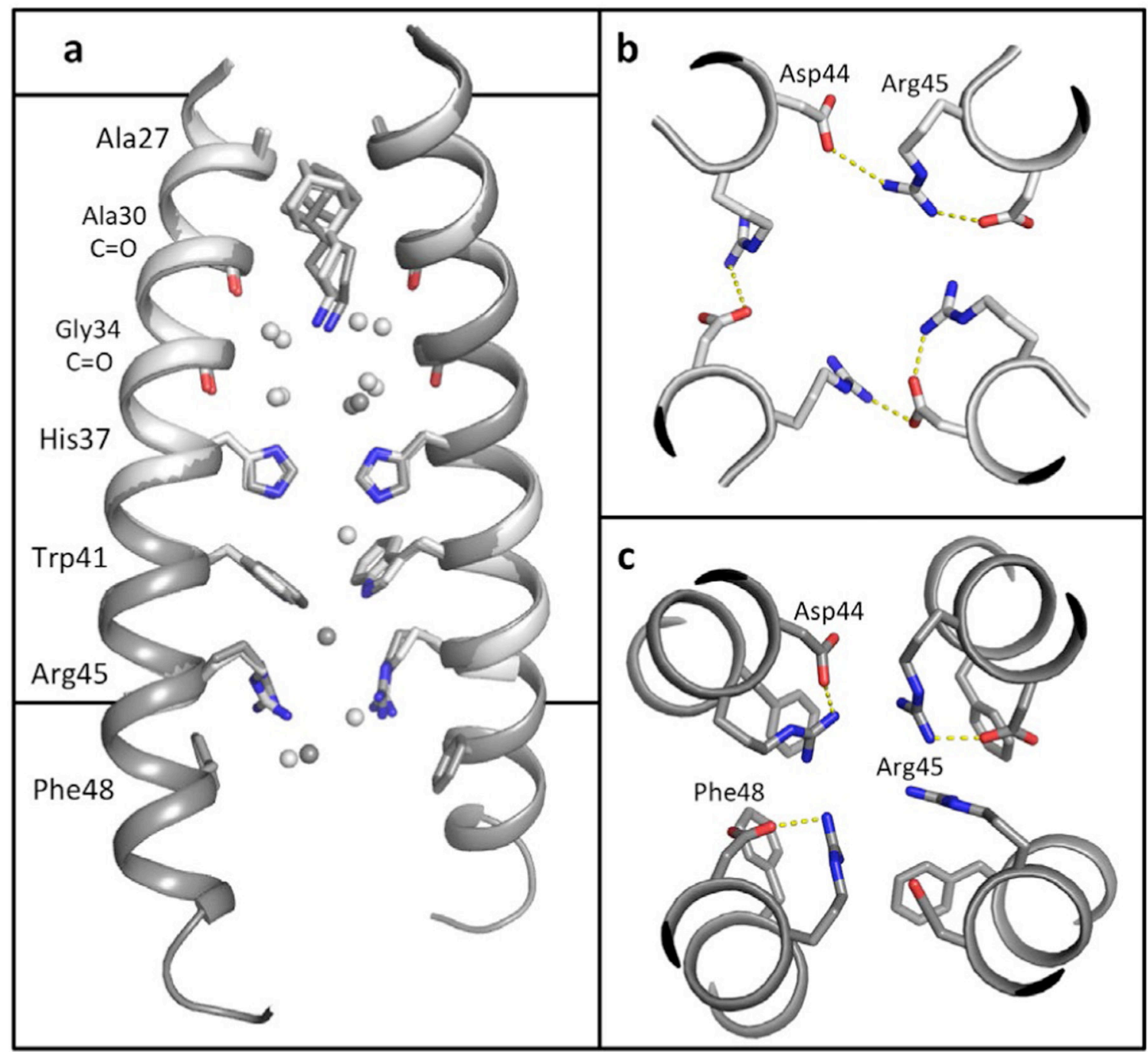

Figure 2.

Structural differences between M2(22-46) V27A (6NV1, light gray) and M2(21-61) V27A (6OUG, dark gray). a. Alignment of 6NV1 and 6OUG. Two monomer helices are shown here (chains $\mathrm{B}$ and D). The binding site of the spiro-adamantyl amine inhibitor is nearly identical in the two structures, though fewer ordered waters are observed in the channel pore in structure 6OUG. Residues 47-55 of M2(21-61) form an a-helix that extends straight downward from the TM helix; the conformational state we observe here is potentially influenced by crystal contacts or choice of lipid mimetic. b. Top-down view of residues Asp44 and Arg45 in the structure of M2(22-46). c. Top-down view of residues Asp44, Arg45, and Phe48 in the structure of M2(21-60). The packing of the channel at the Cterminus is tighter in the longer construct of $\mathrm{M} 2$. 

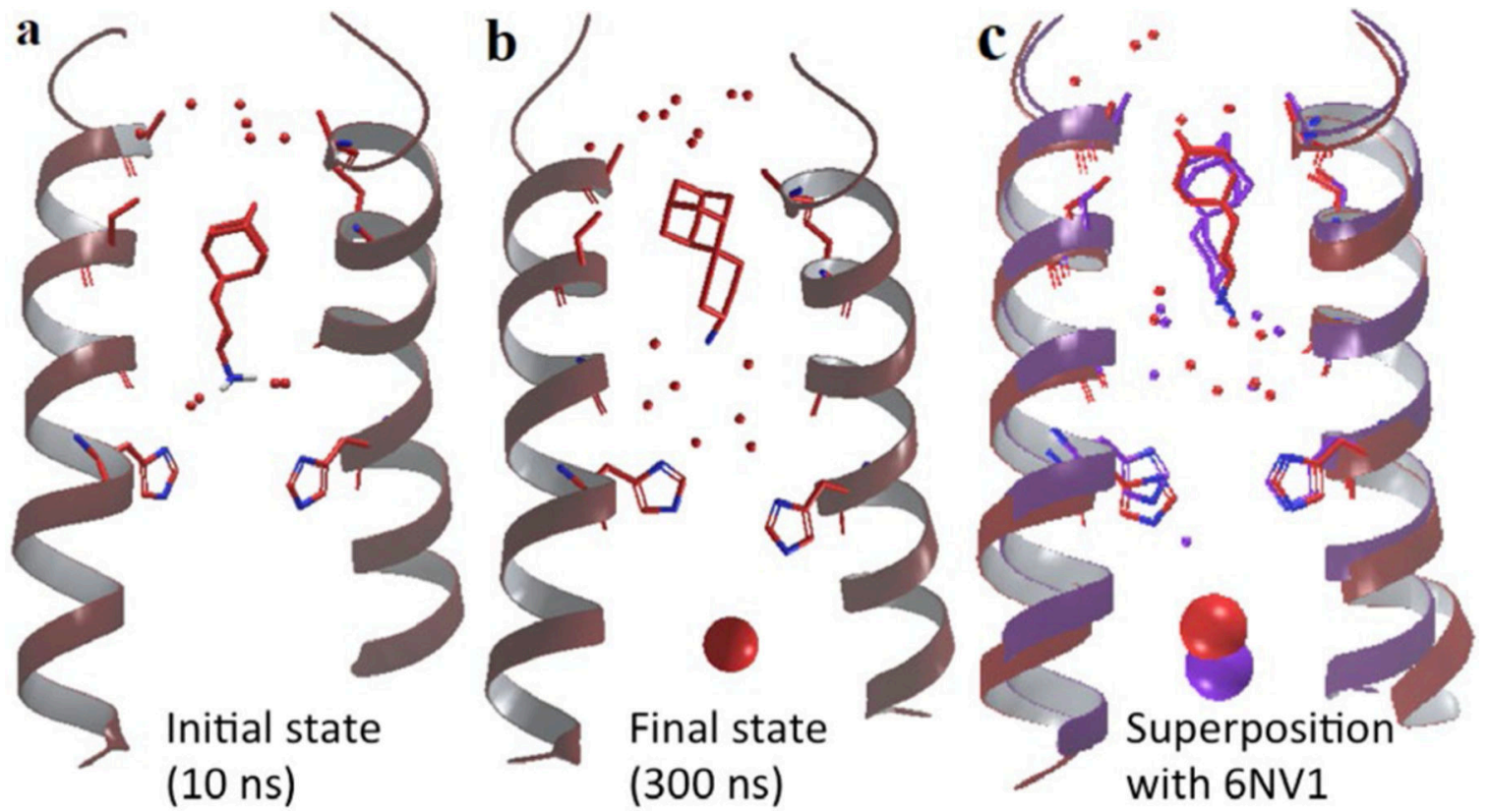

Figure 3.

a. Initial state after $10 \mathrm{~ns}$ of MD simulation of M2 (22-46) V27A - spiro-adamantyl amine complex in a hydrated POPC bilayer. b. Final state after 300 ns of MD simulation. Superposition of spiro-adamantyl amine - M2 (22-46) V27A complexes from X-ray structure (in purple) and $300 \mathrm{~ns}$ MD simulation (in red). The chloride ion at the C-terminus of the channel is shown as a sphere. 